\title{
PRICING OPTIONS ON RISKY ASSETS IN A STOCHASTIC INTEREST RATE ECONOMY ${ }^{1}$
}

\author{
KAUSHIK I. AMIN \\ School of Business Administration, University of Michigan, Ann Arbor, MI \\ ROBERT A. JARROW \\ S. C. Johnson Graduate School of Management, Cornell University, Ithaca, NY
}

\begin{abstract}
This paper studies contingent claim valuation of risky assets in a stochastic interest rate economy. The model employed generalizes the approach utilized by Heath, Jarrow, and Morton (1992) by imbedding their stochastic interest rate economy into one containing an arbitrary number of additional risky assets. We derive closed form formulae for certain types of European options in this context, notably call and put options on risky assets, forward contracts, and futures contracts. We also value American contingent claims whose payoffs are permitted to be general functions of both the term structure and asset prices generalizing Bensoussan (1984) and Karatzas (1988) in this regard. Here, we provide an example where an American call's value is well defined, yet there does not exist an optimal trading strategy which attains this value. Furthermore, this example is not pathological as it is a generalization of Roll's (1977) formula for a call option on a stock that pays discrete dividends.
\end{abstract}

KEyworDs: American call valuation, option pricing, stochastic interest rates, martingale measures

\section{INTRODUCTION}

This paper makes three contributions to the literature on the arbitrage-free pricing of contingent claims. The first contribution is to generalize Heath, Jarrow, and Morton's (1992) interest rate option pricing model to include additional risky assets. This extension enables the HJM methodology to be utilized, for example, to price options on common stocks under stochastic interest rates (generalizing Merton 1973) or options on futures (generalizing Black 1976 and Jarrow 1987). To illustrate these procedures, closed form solutions for European type call and put options on risky assets, forward contracts, and futures contracts are provided.

The second contribution of this paper is to extend HJM (1992) to incorporate Americantype options. Consequently, this analysis generalizes Bensoussan (1984) and Karatzas (1988) to unbounded interest rate processes and claims whose payoffs are dependent on the term structure of interest rates. Finally, the third contribution of this paper is to provide an example of a discontinuous sample path price process where the American call's value is well defined but where there exists no trading strategy attaining this value. This example highlights the important role that sample path continuity of the price process plays in the existing literature on American claim valuation. This example is also of independent interest as it generalizes Roll (1977).

\footnotetext{
${ }^{1}$ This paper includes the content from earlier papers by K. Amin, "Pricing American Options in a Term Structure Economy," 1989, and R. Jarrow, "Option Valuation of Risky Assets in a Stochastic Interest Rate Economy," 1988. Helpful comments from Robin Brenner, Peter Carr, David Heath, and the participants of the Finance Workshop at Cornell University are gratefully acknowledged.

Manuscript received May 1991 ; final version received May 1992.
} 
An outline for this paper is as follows. Section 2 reviews the HJM economy. Section 3 extends this economy to include trading in an arbitrary number of risky assets. Section 4 prices American-type contingent claims, Section 5 provides the example demonstrating the nonexistence of optimal trading strategies, and Section 6 concludes the paper.

\section{THE TERM STRUCTURE MODEL}

This section briefly reviews the HJM (1992) setup for pricing interest rate options. Consider a probability space $(\Omega, F, Q)$ with an augmented Brownian filtration $\left\{F_{t}: t \in[0, \tau]\right\}$ generated by an $n$-dimensional Brownian motion $\left\{W_{1}(t), \ldots, W_{n}(t): t \in[0, \tau] ; \tau<\infty\right\}$ initialized at zero. Let the trading interval be $[0, \tau]$ and define $\lambda$ as the Lebesgue measure on the Borel subsets of $[0, \tau]$. Define $f(t, T), 0 \leq t \leq T \leq \tau$, to be the forward rate contracted at time $t$ for instantaneous borrowing and lending at time $T$.

AsSUMPTION 2.1. Given an initial forward rate curve $\{f(0, T): T \in[0, \tau]\}$, forward rates satisfy the following stochastic integral equation:

$$
\begin{aligned}
f(t, T)= & f(0, T)+\int_{0}^{t} \alpha(u, T, \omega) d u \\
& +\sum_{i=1}^{n} \int_{0}^{t} \sigma_{i}(u, T, \omega) d W_{i}(u) \quad \text { for all } 0 \leq t \leq T \text { and } 0 \leq T \leq \tau,
\end{aligned}
$$

where $\alpha(t, T, \omega)$ and $\sigma_{i}(t, T, \omega)$ for $i=1, \ldots, n$ are assumed to be adapted with respect to $F_{t}$ and jointly measurable and uniformly bounded on $\{(t, v): 0 \leq t \leq v \leq T\}$ $\times \Omega$.

Define an accumulation factor (corresponding to a continuously rolled over money market account) by $B(t)=\exp \left[\int_{0}^{t} r(y) d y\right]$, where $r(y)$ equals the instantaneous spot rate at time $y$, i.e., $r(y)=f(y, y)$.

Next, let $P(t, T)$ be the time $t$ price of a pure discount bond paying $\$ 1$ at time $T(T \geq t)$. By definition of the forward rates,

$$
P(t, T)=\exp \left[-\int_{t}^{T} f(t, v) d v\right] \quad \text { for all } T \in[0, \tau], \quad t \in[0, T]
$$

The discounted value of the $T$-maturity bond is

$$
Z(t, T)=P(t, T) / B(t)
$$

Applying Ito's lemma to $Z(t, T)$ (for details see HJM 1992),

$$
\begin{aligned}
d Z(t, T)= & b(t, T) Z(t, T) d t \\
& +\sum_{i=1}^{n} a_{i}(t, T) Z(t, T) d W_{i}(t) \quad \text { for all } T \in[0, \tau], \quad t \in[0, T]
\end{aligned}
$$


where

$$
a_{i}(t, T)=-\int_{t}^{T} \sigma_{i}(t, u, \omega) d u \quad \text { for } i=1, \ldots, n
$$

and

$$
b(t, T)=-\int_{t}^{T} \alpha(t, u) d u+\frac{1}{2} \sum_{i=1}^{n}\left[\int_{t}^{T} \sigma_{i}(t, u, f(t, u)) d u\right]^{2}
$$

Given Assumption 2.1, $a_{i}(t, T)$ for $i=1, \ldots, n$ and $b(t, T)$ are uniformly bounded on $[0, \tau] \times[0, \tau] \times \Omega$.

This completes the setup of the term structure component of our economy.

\section{THE EXPANDED RISKY ASSET ECONOMY}

This section extends the previous term structure economy to include trading in an arbitrary number of risky assets. We do not restrict our asset set to be finite as in Bensoussan (1984), Karatzas (1988), or Merton (1973). This added level of generality is needed, for example, to price foreign currency options where an infinite number of foreign bonds must be considered (see Amin and Jarrow 1991). We enlarge the previous economy to include $d-n$ additional independent Brownian motions where $\infty>d>n$. The new probability space is $(\Omega, G, Q)$ where $\left\{G_{t}: t \in[0, \tau]\right\}$ is the augmented filtration generated by the $d$-dimensional standard Brownian motion $\left\{W_{1}(t), \ldots, W_{d}(t): t \in[0, \tau]\right\}$ initialized at zero.

Let $X$ be the arbitrary index set of asset types in the economy with $x$ and $y \in X$ denoting generic elements. Let $S(t, x, \omega)$ be the price at time $t$ of asset type $x \in X$ under state $\omega \in \Omega$.

Assumption 3.1. Trading takes place continuously in time and there are no transaction costs, taxes, restrictions on short selling or "other market imperfections" in the economy.

ASSUMPTION 3.2. Risky asset prices satisfy the stochastic integral equation

$$
\begin{aligned}
S(t, x, \omega) & =S(0, x) \exp \left[\int_{0}^{t}(\mu(v, x, \omega)-s(v, x, \omega)\right. \\
& \left.\left.-\left(\frac{1}{2} \sum_{i=1}^{d} \delta_{i}^{2}(v, x, \omega)\right)\right) d v+\sum_{i=1}^{d} \int_{0}^{t} \delta_{i}(v, x, \omega) d W_{i}(v)\right] \text { a.e. } Q
\end{aligned}
$$

where $s(t, x, \omega), \mu(t, x, \omega)$, and $\delta_{i}(t, x, \omega)$ for $i=1, \ldots, d$ are $G_{t}$-adapted, jointly measurable in $(t, \omega) \in[0, \tau] \times \Omega$ and uniformly bounded in $(t, x, \omega) \in[0, \tau] \times X$ $\times \Omega$, and $E\left[\int_{0}^{\tau}|\mu(t, x, \omega)-s(t, x, \omega)|^{2} d t\right]<\infty$ for all $x \in X$.

Given Assumption 3.2, (3.1) can be written as the solution to

$$
\begin{aligned}
d S(t, x, \omega)=[\mu(t, x, \omega)-s(t, x, \omega)] S(t, & x, \omega) d t \\
& +\sum_{i=1}^{d} \delta_{i}(t, x, \omega) S(t, x, \omega) d W_{i}(t) .
\end{aligned}
$$


Here, $s(t, x, \omega)$ represents the dividend rate at time $t$ for asset $x$. Its sign is unrestricted. Next, consider an individual who owns the asset and continuously reinvests his dividends $(s(t, x, \omega))$ by purchasing additional units of the asset. Define the stochastic process $Z(t$, $x$ ) to represent the discounted value of these accumulated holdings, i.e.,

$$
Z(t, x)=\frac{S(t, x)}{B(t)} \exp \left[\int_{0}^{t} s(v, x) d v\right]
$$

This stochastic process satisfies

$$
d Z(t, x)=Z(t, x)\left[\sum_{i=1}^{d} \delta_{i}(t, x) d W_{i}(t)+[\mu(t, x)-r(t)] d t\right] .
$$

Fix $d-n$ assets indexed by $x_{j}$ for $j=1, \ldots,(d-n)$ and consider $n$ bonds of maturities $T_{i}$ for $i=1, \ldots, n$, where $0<T_{1}<T_{2}<\cdots<T_{n} \leq \tau$. From a theoretical perspective, however, we could have chosen any $d$ assets (and no bonds). We want to find a probability measure $\tilde{Q}$ (if it exists) which is equivalent to $Q$ such that $Z\left(t, x_{j}\right)$ and $Z(t$, $\left.T_{i}\right)$ are $\tilde{Q}$-martingales for $j=1, \ldots,(d-n)$ and $i=1, \ldots, n$.

Define

$$
A_{1}=\left[\begin{array}{l}
b\left(t, T_{1}\right) \\
\vdots \\
b\left(t, T_{n}\right) \\
{\left[\mu\left(t, x_{1}\right)-r(t)\right]} \\
\vdots \\
{\left[\mu\left(t, x_{d-n}\right)-r(t)\right]}
\end{array}\right]
$$

and

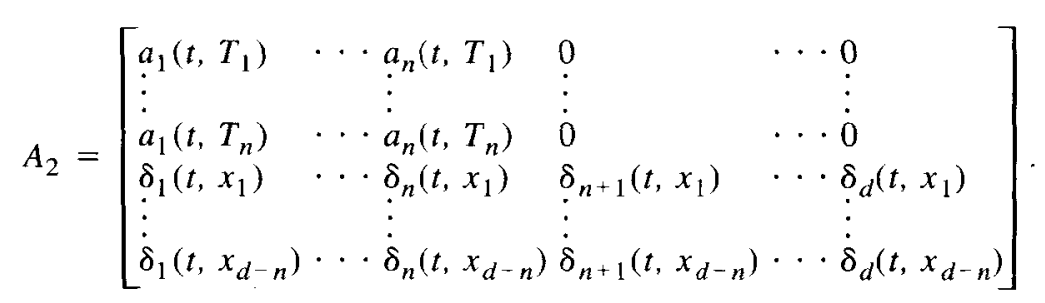

Assumption 3.3. $A_{2}$ is nonsingular a.e. $\lambda \times Q$.

Define $\eta\left(t, T_{1}, \ldots, T_{n} ; x_{1}, \ldots, x_{d-n}\right)$ a $d$-dimensional vector, to be the solution to

$$
A_{1}+A_{2} \eta=0
$$

on the set $\Omega$ where it exists, and define it to be zero on the (null measurable) set where it does not. Given Assumption 3.3, the solution to (3.6) is unique (modulo modifications). Equation (3.6) implies that

$$
\mu(t, x)-r(t)=-\sum_{i=1}^{d} \delta_{i}(t, x) \eta_{i}(t) \text { a.e. } Q .
$$


Hence, the "excess" return on each risky asset is proportional to its variance component with each $\eta_{i}$ the proportionality factor. The components of $\eta$ are termed the market prices of risk corresponding to the sources of randomness in the economy.

Examining the solution to (3.6) reveals that the first $n$ components $\left(\eta_{1}, \ldots, \eta_{n}\right)$ of $\eta$ are identical to those in HJM (1992). A sufficient condition in HJM to guarantee the existence of forward rate processes consistent with no arbitrage is that $\left(\eta_{1}, \ldots, \eta_{n}\right)$ are bounded. As Bensoussan (1984) and Karatzas (1988) also require this assumption, we impose

Assumption 3.4. $\eta_{i}\left(v, T_{1}, \ldots, T_{n} ; x_{1}, \ldots, x_{d-n}\right)$ for $i=1, \ldots, d$ are uniformly bounded on $(v, \omega) \in[0, \tau] \times \Omega$

An immediate consequence is that $(\mu(t, x, \omega)-r(t))$ is uniformly bounded for every $x \in\left\{x_{1}, x_{2}, \ldots, x_{d-n}\right\}$ on $[0, \tau] \times \Omega$. It is now clear why one can not assume that $\mu$ is bounded as in Karatzas [1988] and Bensoussan [1984]. Indeed, as $r$ is unbounded, one must allow $\mu$ to be unbounded.

Proposition 3.1. Under Assumptions (3.1)-(3.4), there exists an equivalent probability measure $\tilde{Q}_{T_{1} \ldots, T_{n} ; x_{1} \ldots, x_{n-d}}$ such that $Z\left(t, x_{j}\right)$ and $Z\left(t, T_{i}\right)$ are martingales with respect to $\left\{G_{t}: 0 \leq t \leq \tau\right\}$ for $j=1, \ldots,(d-n)$ and $i=1, \ldots, n$.

Proof. Let $\tilde{Q} \equiv \tilde{Q}_{T_{1}, \ldots, T_{n} ; x_{1}, \ldots, x_{n-d}}$ be a probability measure on $(\Omega, G)$ which is equivalent to $Q$ and is defined by

$$
\begin{aligned}
\frac{d \tilde{Q}}{d Q}=\exp \left(\sum_{i=1}^{d} \int_{0}^{T_{1}} \eta_{i}\left(v, T_{1}, \ldots, T_{n} ; x_{1}, \ldots, x_{d-n}\right) d W_{i}(v)\right. \\
\left.\quad-\frac{1}{2} \sum_{i=1}^{d} \int_{0}^{T_{1}} \eta_{i}^{2}\left(v, T_{1}, \ldots, T_{n} ; x_{1}, \ldots, x_{d-n}\right) d v\right)
\end{aligned}
$$

Let $\tilde{E}$ be the expectation w.r.t. $\tilde{Q}$. By Girsanov's theorem,

$$
\tilde{W}_{i}(t)=W_{i}(t)-\int_{0}^{t} \eta_{i} d v \quad \text { for } i=1, \ldots, d,
$$

is a $d$-dimensional Brownian motion with respect to $\tilde{Q}$.

Defining $\bar{Z}(t) \equiv\left[Z\left(t, T_{1}\right), \ldots, Z\left(t, T_{n}\right) ; Z\left(t, x_{1}\right), \ldots, Z\left(t, x_{d-n}\right)\right]^{\prime}$ and substituting (3.9) into (2.4) and (3.4), we can write the vector equation

$$
\begin{aligned}
& d \bar{Z}=\bar{Z}\left[A_{1} d t+A_{2} d W(t)\right], \\
& d \bar{Z}=\bar{Z}\left[A_{2} d \tilde{W}(t)\right]
\end{aligned}
$$

Since $\left\|A_{2}\right\|$ is bounded, Novikov's condition implies $\bar{Z}(t)$ is a $\tilde{Q}$-martingale.

Proposition 3.2. The equivalent martingale measure identified in Proposition 3.1 is unique. 
Proof. If $\bar{Q}$ is another probability measure on $(\Omega, G)$, which is equivalent to $Q$, and under which $\bar{Z}(t)$ is a martingale with respect to $G_{t}$, then define $\beta=d \bar{Q} / d Q$. By Liptser and Shiryayev $\left(1977\right.$, p. 234) there exist $G_{t}$-adapted processes $Y_{j}(t)$ for $j=1, \ldots, d$, such that $\int_{0}^{\tau}\left(Y_{j}(t)\right)^{2} d t<\infty$ a.e. $\bar{Q}$ and

$$
\beta(t)=\beta(0)+\sum_{j=1}^{d} \int_{0}^{t} Y_{j}(v) d W_{j}(v) \quad \text { for all } 0<t<\tau,
$$

where $\beta(t)=E\left[\beta \mid G_{t}\right]$. Now let $\zeta_{j}(v)=\beta^{-1}(v) Y_{j}(v)$. Using Liptser and Shiryayev (1977, Theorem 6.4, p. 234), $\bar{W}_{j}(t)=W_{j}(t)-\int_{0}^{t} \zeta_{j}(v) d v$ is a $d$-dimensional Brownian motion with respect to $\bar{Q}$. Substituting this into (3.10) and rearranging, we get

$$
d \bar{Z}=\bar{Z}\left[\left[A_{1}+A_{2} \zeta\right] d t+A_{2} d \bar{W}_{t}\right]
$$

Now, $\bar{Z}$ is a $\bar{Q}$-martingale by hypothesis. This implies that the drift term must be a.s. zero in (3.16). Therefore, $\zeta=\eta$ a.s. $Q$ because the solution to $A_{1}+A_{2} \zeta=0$ is a.s. unique. Hence we obtain the equivalence of $\bar{Q}$ and $\tilde{Q}$.

The martingale measure in Proposition 3.2 depends upon the assets $T_{1}, \ldots, T_{n}$ and $x_{1}, \ldots, x_{d^{-} n}$. To obtain a martingale measure independent of the assets selected, we add

Assumption 3.5. $\eta_{k}\left(t, T_{1}, \ldots, T_{n} ; x_{1} \ldots, x_{d-n}\right)$ is independent of $T_{1}, \ldots, T_{n}$; $x_{1}, \ldots, x_{d-n}$ for each $t \in[0, \tau]$ and $k=1, \ldots, d$.

Henceforth, we will denote the market prices of risk by the vector $\phi(t)=\left(\phi_{1}(t), \ldots\right.$, $\left.\phi_{d}(t)\right)$ and write $\tilde{Q}_{T_{1}, \ldots, T_{n} ; x_{1}, \ldots, x_{n-d}} \equiv \tilde{Q}$. Brennan and Schwartz (1979), implicitly, and HJM (1992), explicitly, require this assumption to obtain their pricing relationships across all maturity bonds.

Using Harrison and Pliska (1981) or the analysis in Section 4, we can now price European options as the expected value, under $\tilde{Q}$, of the discounted payoffs to the option at maturity. This present value operator is often called the "risk-neutral" operator. To illustrate this procedure, we provide an example.

EXAMPLE (Merton 1973). This example corresponds to an extension of the economy analyzed by Merton (1973) to include a random evolution of the entire term structure of interest rates. We provide explicit solutions for the values of European call options on a risky asset, a forward contract, and a futures contract. The corresponding put option formulae are obtainable by put-call parity.

We consider a case where $n=1$ and $d=2$. The forward rate process, satisfying Assumption 2.1, is given by

$$
d f(t, T)=\alpha(t, T, \omega) d t+\sigma(t, T) d W_{1}(t)
$$

where $\sigma:\{(u, v): 0 \leq u \leq v \leq \tau\} \rightarrow R$ is a bounded, deterministic, strictly nonzero function. 
The risky asset satisfies

$$
d S(t)=\mu S(t) d t+\delta_{1} S(t) d W_{1}(t)+\delta_{2} S(t) d W_{2}(t)
$$

where $\delta_{1}, \delta_{2}$, and $S(0)>0$ are positive constants and $\mu$ satisfies the conditions in Assumption 3.2 .

It is easy to see that Assumption 3.2 and 3.3 are satisfied. Define $\eta_{1}(t), \eta_{2}(t)$ by

$$
\left[\begin{array}{l}
b(t, T) \\
\mu-r(t)
\end{array}\right]+\left[\begin{array}{ll}
a(t, T) & 0 \\
\delta_{1} & \delta_{1}
\end{array}\right]\left[\begin{array}{l}
\eta_{1}(t) \\
\eta_{2}(t)
\end{array}\right]=\left[\begin{array}{l}
0 \\
0
\end{array}\right]
$$

where

$$
a(t, T) \equiv-\int_{t}^{T} \sigma(t, v) d v \quad \text { and } \quad b(t, T) \equiv-\int_{t}^{T} \alpha(t, v) d v+\frac{1}{2}\left[\int_{t}^{T} \sigma(t, v) d v\right]^{2}
$$

We assume that the conditions of Assumptions 3.4 and 3.5 hold. By Proposition 3.1, there exists a probability measure $\tilde{Q}$ on $\left\{(\Omega, G),\left\{G_{t}: t \in[0, \tau]\right\}\right\}$ making $Z(t, T)=P(t$, $T) / B(t)$ and $z(t)=S(t) / B(t) \tilde{Q}$-martingales. Note that

$$
\left[\begin{array}{l}
d Z(t, T) / Z(t, T) \\
d z(t) / z(t)
\end{array}\right]=\left[\begin{array}{cc}
a(t, T) & 0 \\
\delta_{1} & \delta_{2}
\end{array}\right]\left[\begin{array}{l}
d \tilde{W}_{1}(t) \\
d \tilde{W}_{2}(t)
\end{array}\right]
$$

where

$$
\tilde{W}_{i}(t)=W_{i}(t)-\int_{0}^{t} \eta_{i}(v) d v \quad \text { for } i=1,2
$$

are Brownian motions with respect to $\bar{Q}$. Our goal is to write out explicit representations of $B(t), S(t)$, and $P(t, T)$ in terms of the parameters of the system. It can be shown that (see HJM 1992)

$$
B(t)=\frac{1}{P(0, t)} \exp \left\{\frac{1}{2} \int_{0}^{t} a(s, t)^{2} d s-\int_{0}^{t} a(s, t) d \tilde{W}_{1}(s)\right\}
$$

where $\int_{0}^{t} a(s, t) d \tilde{W}_{1}(s)$ is normal $\left(0, \int_{0}^{t} a(s, t)^{2} d s\right)$ under $\bar{Q}$. Further, tedious algebra yields

$$
S(t)=S(0) B(t) \exp \left\{-\frac{1}{2}\left[\delta_{1}^{2}+\delta_{2}^{2}\right] t+\delta_{1} \tilde{W}_{1}(t)+\delta_{2} \tilde{W}_{2}(t)\right\},
$$

where $\delta_{1} \tilde{W}_{1}(t)+\delta_{2} \bar{W}_{2}(t)$ is normal $\left(0,\left[\delta_{1}^{2}+\delta_{2}^{2}\right] t\right)$ and

$$
\begin{aligned}
P(t, T)=\frac{P(0, T)}{P(0, t)} \exp \left\{\int_{t}^{T} \int_{0}^{t} \sigma(y, s) a(y, s) d y d s\right. \\
\left.-\int_{0}^{t}\left[\int_{t}^{T} \sigma(y, s) d s\right] d \tilde{W}_{1}(y)\right\},
\end{aligned}
$$


where $\int_{0}^{t}\left[\int_{t}^{T} \sigma(y, s) d s\right] d \tilde{W}_{1}(y)$ is normal $\left(0, \int_{0}^{t}\left[\int_{t}^{T} \sigma(y, s) d s\right]^{2} d y\right)$ under $\tilde{Q}$.

Consider a European call option on the risky asset $S(t)$ with an exercise price of $K$ and a maturity date $T<\tau$. The call's value at time 0 is:

$$
C(0)=\tilde{E}(\max (S(T)-K, 0) / B(T)) .
$$

A calculation yields

$$
C(0)=S(0) \Phi(h)-K P(0, T) \Phi(h-\psi),
$$

where

$$
\begin{aligned}
h & =\frac{\log (S(0) / K P(0, T))+\frac{1}{2} \psi^{2}}{\psi}, \\
\psi^{2} & =\left[\delta_{1}^{2}+\delta_{2}^{2}\right] T-2 \delta_{1} \int_{0}^{T} a(t, T) d t+\int_{0}^{T} a(t, T)^{2} d t
\end{aligned}
$$

and $\Phi(\cdot)$ is the cumulative normal distribution. This is Merton's (1973) call option formula for a bond price process given by $(3.20)$.

Now consider forward and futures contracts on this risky asset. It is easy to show that the forward price, $K(t)$, at time $t$, corresponding to contract maturity $\tau$, is given by

$$
K(t)=S(t) / P(t, \tau)
$$

The value of a European call option, with maturity date $\tau$ and exercise price $M>0$, on this forward contract is

$$
C_{K}(0)=\tilde{E}(\max (K(T)-M, 0) / B(T)) .
$$

Substituting (3.23) and the expressions for $S(T), P(T, \tau)$, and $B(T)$ yields

$$
C_{K}(0)=S(0) \frac{P(0, T)}{P(0, \tau)} e^{\xi} \Phi(h)-M P(0, T) \Phi(h-\eta),
$$

where

$$
\begin{aligned}
\xi= & \delta_{1} \int_{0}^{T}\left[\int_{T}^{\tau} \sigma(y, v) d v\right] d y+\frac{1}{2} \int_{0}^{T}\left[\int_{T}^{\tau} \sigma(y, v) d v\right]^{2} d y \\
& -\int_{T}^{\tau} \int_{0}^{T} \sigma(y, v) a(y, v) d y d v, \\
h= & \frac{1}{\eta}\left[\log \left(\frac{S(0) e^{\xi}}{P(0, \tau) M}\right)+\frac{1}{2} \eta^{2}\right]
\end{aligned}
$$


and

$$
\eta=\int_{0}^{T}\left[\delta_{1}+\int_{T}^{\tau} \sigma(s, v) d v-a(s, t)\right]^{2} d s+\delta_{2}^{2} T
$$

We now consider a futures contract. Again, a standard argument shows that the futures price, $k(t)$, is a $\tilde{Q}$ martingale. Therefore,

$$
k(T)=\tilde{E}\left(S(\tau) \mid G_{T}\right)=\frac{S(T)}{P(T, \tau)} \exp \left[-\delta_{1} \int_{T}^{\tau} a(s, \tau) d s+\int_{T}^{\tau} a(s, \tau)^{2} d s\right]
$$

Given (3.25) for the futures price, (3.23) implies that

$$
k(t)=K(t) \exp \left[-\delta_{1} \int_{t}^{\tau} a(s, \tau) d s+\int_{t}^{\tau} a(s, \tau)^{2} d s\right] .
$$

The futures price equals the forward price times an adjustment factor. If $\delta_{1}>0$, the futures prices can exceed the forward price as the adjustment factor, $\exp \left\{-\delta_{1} \int_{t}^{\tau} a(s, t)\right.$ $\left.d s+\int_{t}^{t} a(s, t)^{2} d s\right\}$ will be greater than 1 since $a(s, t)=-\int_{s}^{t} \sigma(s, v) d v<0$.

A European option on the futures contract with a maturity date $T<\tau$ and exercise price $M$ has a terminal payoff equal to $C_{k}(T)=\max (k(T)-M, 0)$. Thus,

$$
\begin{aligned}
& C_{k}(0)=\tilde{E}\left(\frac{\max (k(T)-M, 0)}{B(T)}\right) \\
&=\tilde{E}\left(\operatorname { m a x } \left(\frac { S ( T ) } { P ( T , \tau ) } \operatorname { e x p } \left[-\delta_{1} \int_{T}^{\tau} a(s, \tau) d s\right.\right.\right. \\
&\left.\left.\left.+\int_{T}^{r} a(s, \tau)^{2} d s\right]-M, 0\right) / B(T)\right) .
\end{aligned}
$$

Substitution of the appropriate expressions yields

$$
C_{k}(0)=S(0) \frac{P(0, T)}{P(0, \tau)} e^{\xi+\lambda} \Phi(h)-M P(0, T) \Phi(h-\eta)
$$

where

$$
\begin{aligned}
\xi= & \delta_{1} \int_{0}^{T}\left[\int_{T}^{\tau} \sigma(y, v) d v\right] d y+\frac{1}{2} \int_{0}^{T}\left[\int_{T}^{\tau} \sigma(y, v) d v\right]^{2} d y \\
& -\int_{T}^{\tau} \int_{0}^{T} \sigma(y, v) a(y, v) d y d v, \\
\lambda= & -\delta_{1} \int_{T}^{\tau} a(s, \tau) d s+\int_{T}^{\tau} a(s, \tau)^{2} d s, \\
h= & \frac{1}{\eta}\left[\log \left(\frac{S(0) e^{\xi+\lambda}}{P(0, \tau) M}\right)+(1 / 2) \eta^{2}\right],
\end{aligned}
$$


and

$$
\eta=\int_{0}^{T}\left[\delta_{1}+\int_{T}^{\tau} \sigma(s, v) d v-a(s, t)\right]^{2} d s+\delta_{2}^{2} T
$$

This valuation formula differs from that of a forward option through the parameter $\lambda$, which is positive if $\delta_{1}>0$ (since $a(s, t)=-\int_{s}^{t} \sigma(s, v) d v$ ), so that $C_{K}(0)<C_{k}(0)$. Interest rates being nonstochastic (i.e., $\sigma(s, v) \equiv 0$ ) is a sufficient condition for this formula to collapse to that of the forward option. This completes the example.

\section{VALUING AMERICAN CONTINGENT CLAIMS}

To extend the previous model to value American contingent claims we need to investigate the concept of a "trading strategy."

Definition 4.1. An American contingent claim is a triplet $\left(\tau_{M}, c, Y\right)$ consisting of

(i) An expiration date $\tau_{M} \in(0, \tau]$.

(ii) A continuous, nonnegative, $G_{t}$-adapted cash flow $c(t)$ per unit time on $\left[0, \tau_{M}\right]$.

(iii) The selection of an exercise date $\theta$ and a continuous, nonnegative, $G_{t}$-adapted payoff $Y(\theta) . Y(\theta)$ is the reward on the exercise date $\theta$.

The exercise date $\theta$ is chosen from $\theta \in \tau_{\left(0, \tau_{M}\right]}$, where $\tau_{\left(0, \tau_{M}\right]}$ is the class of all stopping times in $\left(0, \tau_{M}\right]$. As written, an American contingent claims has intermediate cash flows. To simplify the mathematics, we can transform this American contingent claim into one without intermediate cash flows by reinvesting the intermediate cash flows into a fund (a money market account) which earns the riskless rate. We will denote the amount of wealth invested in this fund at time $t$ to be $F(t)$. Last, we require that only additions (and no withdrawals) be allowed from this fund as the cash flows are assumed to be nonnegative.

The running discounted payoff from the contingent claim $\left(\tau_{M}, c, Y\right)$ is then the discounted accumulated payoff from the claim if exercised at time $t$, i.e.,

$$
U(t)=\frac{Y(t)}{B(t)}+\int_{0}^{t} \frac{c(v)}{B(v)} d v
$$

By construction, $U(t)$ is a continuous, nonnegative, and $G_{t}$-adapted process on $\left[0, \tau_{M}\right]$.

We will price claims which satisfy

$$
E\left[\left(\sup _{t \in\left[0, \tau_{M}\right]} U(t)\right)^{p}\right]<\infty \quad \text { for some } p>1 .
$$

It can be shown that the common types of options (calls and puts) satisfy this assumption. This assumption is different from that employed by either Bensoussan (1984) or Karatzas (1988). An equivalent version of this assumption under the martingale measure is needed later. To obtain this version, we require a lemma. 
LEMmA 4.1. Given a nonnegative random variable $R \in G_{\tau_{M}}$ with $E\left(R^{p}\right)<\infty$ for $p$ $>1$, then $\tilde{E}\left(R^{q}\right)<\infty$, where $q=\sqrt{p}>1$.

Proof. By the Radon-Nikodym theorem

$$
\tilde{E}\left(R^{q}\right)=E\left[R^{q} N\left(\tau_{M}\right)\right],
$$

where

$$
N(t)=E\left(\frac{d \tilde{Q}}{d Q} \mid G_{t}\right)
$$

An application of Ito's lemma confirms that

$$
\begin{aligned}
{\left[N\left(\tau_{M}\right)\right]^{h}=} & \exp \left[\sum_{i=1}^{d} \int_{0}^{\tau_{M}} h \eta_{i}(t) d W_{i}(t)-\sum_{i=1}^{d} \int_{0}^{\tau_{M}} \frac{1}{2} h^{2} \eta_{i}^{2}(t) d t\right] \\
& \times \exp \left[\sum_{i=1}^{d} \int_{0}^{\tau_{M}} \frac{1}{2}\left(h^{2}-h\right) \eta_{i}^{2}(t) d t\right]
\end{aligned}
$$

where $h$ satisfies $1 / q+1 / h=1$. Using the boundedness of $\eta_{i}$ and Novikov's theorem, $E\left[N\left(\tau_{M}\right)^{h}\right]<\infty$. Since Holder's inequality gives

$$
E\left[R^{q} N\left(\tau_{M}\right)\right] \leq\left[E\left(R^{p}\right)\right]^{1 / q}\left[E\left(N\left(\tau_{M}\right)^{h}\right)\right]^{1 / h}
$$

and the first factor is finite by hypothesis, we are done.

Lemma 4.1 and expression (4.2) imply

$$
\tilde{E}\left[\sup _{t \in\left[0, \tau_{M}\right]} U(t)\right]^{q}<\infty \quad \text { for some } q>1 .
$$

For ease of notation, we will denote the money market account as asset 0 , the bonds $T_{1}, \ldots, T_{n}$ as assets $1, \ldots, n$ and the risky assets $x_{j}$ for $j=1, \ldots,(d-n)$ as assets $(n+1), \ldots, d$. The prices of these assets will be denoted $S_{j}(t)$ for $j=0, \ldots, d$. To be consistent, note that $s_{j}(t)=0$ for $j=0, \ldots, n$ (dividend rates are zero for these assets). Let $\delta_{i j}(t)$ be the variance coefficient of $W_{i}(t)$ for the $j$ th asset price. Note that by (3.5), $\delta_{i j}(t)=0$ for $i=(n+1), \ldots, d$ and $j=1, \ldots, n$. By the definition of the money market account, $B(t)$, we also have that $\delta_{i 0}(t)=0$ for $i=1, \ldots, d$. Given the previous framework, the $\delta_{i j}(t)$ 's are well defined.

We will also restrict our "trading strategies" to include only these $d$ assets. From the subsequent discussion, it will become clear that we can replicate the payoffs from any other traded asset in the economy using only these assets.

Let $\pi_{j}(t)$ be the number of shares owned of asset $j$ at time $t$ for $j=0, \ldots, d$. A trading strategy $\pi(t)=\left\{\pi_{j}(t): j=0, \ldots, d\right\}$ is a $G_{t}$-adapted process which satisfies

$$
\sum_{i=1}^{d} \int_{0}^{t}\left[\frac{\pi_{j}(t) S_{j}(t)}{B(t)}\right]^{2} \delta_{i j}(t) d t<\infty \quad \text { a.s. } Q \quad \text { for } i=1, \ldots, d
$$


Expression (4.8) is an integrability condition required to define the stochastic integral in (4.10). Next, define $V(t)$ to be the corresponding value process given by

$$
V(t)=\sum_{j=0}^{d} \pi_{j}(t) S_{j}(t) .
$$

DEFINITION 4.2. A trading strategy $\pi$ is admissible and self-financing (s.f.t.s.) is $V(t)$ $\geq 0$ a.e. $Q$ for all $t \in\left[0, \tau_{M}\right]$, and

(i) There exists a nonnegative, right-continuous, $G_{t}$-adapted process $F(t)$, with $F(0)=0$, such that

$$
\begin{aligned}
V(t)+F(t)=V(0)+ & \sum_{j=0}^{d} \int_{0}^{t} \pi_{j}(v) d S_{j}(v)+\sum_{j=0}^{d} \int_{0}^{t} \pi_{j}(v) S_{j}(v) s_{j}(v) d v \\
& +\int_{0}^{t} F(v) r(v) d v \text { a.s. } Q \quad \text { for all } t \in\left[0, \tau_{M}\right],
\end{aligned}
$$

and

(ii) $F(t) / B(t)$ is a.s. nondecreasing on $\left[0, \tau_{M}\right]$, i.e., withdrawals from the fund are not allowed.

Equation (4.10) is the self-financing constraint where the last term incorporates the investment in a fund, $F(t)$, which earns the spot rate. The purpose of this fund is to allow cash inputs into an otherwise self-financing trading strategy. These cash inputs are needed in the construction of a synthetic American contingent claim as we must match any intermediate cash flows received on the American contingent claim (see the discussion below Definition 4.1).

Let us consider an arbitrary self-financing trading strategy $(\pi, F)$. We define the discounted value process (corresponding to the total payoff) of this trading strategy as

$$
V_{D}(t) \equiv \frac{V(t)}{B(t)}+F_{D}(t) \quad \text { where } F_{D}(t) \equiv \frac{F(t)}{B(t)}
$$

By a standard argument (see Karatzas 1988), for any admissible self-financing trading strategy $(\pi, F), V_{D}(t)$ is a $\tilde{Q}$-supermartingale with respect to $G_{t}$. The optional stopping theorem now yields

$$
\tilde{E}\left(V_{D}(t)\right) \leq V(0) \quad \text { for all } t \in\left[0, \tau_{M}\right]
$$

This implies that

$$
\tilde{E}\left(F_{D}(t)\right) \leq V(0) \quad \text { for all } t \in\left[0, \tau_{M}\right]
$$

In other words, the nonnegativity requirement that $V(t) \geq 0$ restricts the amount of money that can be put into the fund to be no greater (in expectation) than the initial value of the trading strategy. 
Definition 4.3. The arbitrage free price for the claim $\left(\tau_{M}, c, Y\right)$ at time $t=0$ is given by $\sup _{\theta \in \tau_{(0, T M)}} \tilde{E}(U(\theta))$.

This definition can be justified by similar arguments as in Karatzas (1988). In the next proposition, we prove that this supremum can be attained by a suitable trading strategy which at all times maintains at least as much wealth in the fund as the cumulative cash flows generated by the contingent claim. Furthermore, the value process from this trading strategy, if exercised, is always greater than or equal to the payoff of the claim.

PROPOSITION 4.1. There exists an admissible self-financing trading strategy with a value process $V(t)$ which satisfies

$$
\frac{V(t)}{B(t)}=\underset{\left.\theta \in \tau_{(t, \tau} \tau_{M}\right)}{\operatorname{ess} \sup } \tilde{E}\left[U(\theta) \mid G_{t}\right] \text { a.e. } \tilde{Q},
$$

and a fund process $F(t)$ such that $F(t) \geq \int_{0}^{t} c(v) \exp \left(\int_{v}^{t} r(u) d u\right) d v$.

Proof. The proof is similar to Karatzas (1988, Theorem 5.4). From Fakeev (1970, Theorem 1), there exists a nonnegative supermartingale $X(t)$ with RCLL paths such that

$$
\sup _{\theta \in \tau_{\left(t, r_{M}\right)}} \tilde{E}[U(\theta)]=\tilde{E}[X(t)]
$$

and

$$
X(t)=\underset{\theta \in \tau_{\left(t, \tau_{M}\right)}}{\operatorname{ess} \sup } \tilde{E}\left[U(\theta) \mid G_{t}\right] \text { a.e. } \bar{Q}
$$

By Theorem 4 in Fakeev (1970), the random variable $\theta(t)=\inf \{v: v \geq t ; X(v)=U(v)\}$ is optimal for the problem. Given that $\bar{E}\left[\sup _{r \in\left[0, \tau_{M}\right]} U(t)\right]^{q}<\infty$ for some $q>1$ (notice that we have proved this under different conditions), we can write (see lemma 5.5 in Karatzas 1988),

$$
X(t)=X(0)+M(t)-\Lambda(t)
$$

where $M(t)$ is a $\bar{Q}$-martingale with $M(0)=0$ and $\Lambda(t)$ is a continuous, nondecreasing process of bounded variation with $\Lambda(0)=0$. Applying a martingale representation theorem (Karatzas and Shreve 1988; p. 184) yields

$$
X(t)=X(0)+\sum_{i=1}^{d} \int_{0}^{t} g_{i}(v) d \tilde{W}_{i}(v)-\Lambda(t),
$$

where $g_{i}(v)$ are adapted processes which satisfy $\int_{0}^{\tau_{M}} g_{i}^{2}(v) d v<\infty$ a.s. $\tilde{Q}$ for $i=1, \ldots$, $d$. Define $\tilde{V}(t)$ as the solution to the following equation:

$$
\left[\frac{\tilde{V}(t)}{B(t)}\right]+\int_{0}^{t} \frac{c(u)}{B(u)} d u=X(t)
$$


Substituting this equation into (4.18) and integrating,

$$
\left[\frac{\tilde{V}(t)}{B(t)}\right]+\int_{0}^{t} \frac{c(u)}{B(u)} d u+\int_{0}^{t} d \Lambda(u)=X(0)+\sum_{i=1}^{d} \int_{0}^{t} g_{i}(v) d \tilde{W}_{i}(v)
$$

Now define $\tilde{F}_{D}(t)(=\tilde{F}(t) / B(t)), \tilde{A}(t)$, and $\tilde{V}_{D}(t)$ by

$$
\begin{gathered}
\tilde{F}_{D}(t)=\int_{0}^{t} d \Lambda(u)+\int_{0}^{t} \frac{c(u)}{B(u)} d u, \\
\frac{\tilde{A}(t)}{B(t)}=\int_{0}^{t} d \Lambda(u), \\
\tilde{F}_{D}(t)=\frac{\tilde{V}(t)}{B(t)}+\tilde{F}_{D}(t) .
\end{gathered}
$$

As $\Lambda(t)$ is nondecreasing, (4.21) implies that $\tilde{F}_{D}(t) \geq \int_{0}^{t} c(v) \exp \left[\int_{0}^{t} r(u) d u\right] d v$. Noting that $X(0)=\tilde{V}(0),(4.20)$ can be rewritten as

$$
\tilde{V}_{D}(t)=\left[\frac{\vec{V}(t)}{B(t)}\right]+\tilde{F}_{D}(t)=\tilde{V}(0)+\sum_{i=1}^{d} \int_{0}^{t} g_{i}(v) d \tilde{W}_{i}(v)
$$

Now define the trading strategy $\pi(t)$ as the solution to

$$
g_{i}(t)=\sum_{j=1}^{d} \frac{\pi_{j}(t) \exp \left[-\int_{0}^{t} s(v) d v\right] S_{j}(t) \delta_{i j}(t)}{B(t)} \quad \text { for } i=1, \ldots, d
$$

and

$$
\pi_{0}(t) B(t)=\tilde{V}(t)-\sum_{j=1}^{d} \pi_{j}(t) S_{j}(t)
$$

As $A_{2}$ is invertible (assumption 3.3), the above system of equations has a unique solution. As $s(v)$ is bounded and $\int_{0}^{\tau M} g_{i}^{2}(v) d v<\infty$ a.s. $\tilde{Q}$, this satisfies the definition of a trading strategy.

Now, substituting (3.11) and (4.25) into (4.24), we get

$$
\tilde{V}_{D}(t)=\tilde{V}(0)+\sum_{j=0}^{d} \int_{0}^{t} \pi_{j}(v) \exp \left[-\int_{0}^{t} s(v) d v\right] d Z_{j}(v)
$$

An application of Ito's lemma shows that this satisfies (4.10). To complete the proof that it is both admissible and self-financing, we only need to show that $\tilde{V}(t) \geq 0$. Theorem 2 in Fakeev (1970) gives us the result that $X(t)$ is the smallest, nonnegative, supermartingale that majorizes $U(t)$. This implies that $X(t) \geq U(t)$ and $X\left(\tau_{M}\right)=U\left(\tau_{M}\right)$. Hence, $\tilde{V}(t) \geq$ $Y(t)$ and $\tilde{V}\left(\tau_{M}\right)=Y\left(\tau_{M}\right)$. In particular, $\tilde{V}(t) \geq 0$ because $Y(t) \geq 0$ by assumption. 
Proposition 4.1 allows us to value any contingent claim satisfying expression (4.2). Unfortunately, no general closed-form solutions to (4.18) are known under stochastic interest rates. Consequently, the standard procedure for determining these values is to resort to numerical approximation techniques.

\section{EXAMPLE: NONEXISTENCE OF OPTIMAL EXERCISE STRATEGIES}

In previous sections, given that the accumulated price process (3.3) is continuous a.e., we have shown both the existence of the American claim's value and an appropriate trading strategy which attains this value. Here we analyze an example where this analysis fails in the presence of a discontinuity. This example generalizes Roll's (1977) formula for an American call option on a stock with known discrete dividends on fixed dates. For this example, the value function exists and can be characterized but there does not exist an optimal "early exercise strategy" nor a trading strategy that generates the claim's value. As Roll's formula is widely used in the academic literature, this example is of considerable interest.

Consider the introduction of an additional stock $S^{*}(t)$ in the economy. Let

$$
S^{*}(t) \equiv S\left(t, x_{d}\right)+d_{1} P\left(t, T^{*}\right) 1_{\left(t<T^{*}\right)}
$$

i.e.,

$$
\begin{aligned}
S^{*}(t)=S\left(0, x_{d}\right) \exp \left[\int_{0}^{t}\left[\mu\left(u, x_{d}\right)-\frac{1}{2} \sum_{i=1}^{d} \delta_{i}^{2}\left(u, x_{d}\right)\right] d u\right. & \\
& \left.\quad+\sum_{i=1}^{d} \int_{0}^{t} \delta_{i}\left(u, x_{d}\right) d W_{i}(u)\right]+d_{1} P\left(t, T^{*}\right) 1_{\left(t<T^{*}\right)},
\end{aligned}
$$

where $d_{1}$ is the fixed dividend paid at date $T^{*}$, and $1\left(t<T^{*}\right)$ is the indicator function equal to one if $t<T^{*}$ and zero otherwise. Note that the stock price is everywhere rightcontinuous, but discontinuous from the left at $t=T^{*}$. The assets $S^{*}$ can be viewed as a portfolio consisting of the risky asset $S\left(t, x_{d}\right)$ which does not pay any dividends and $d_{1}$ bonds with maturity $T^{*}$. With this insight, it will be easier to understand the subsequent manipulations. At this stage, we add one additional assumption.

Assumption 5.1. Nonnegative interest rates and no capital losses on zero coupon bonds:

(i) $Q(B(t+\varepsilon) \geq B(t))=1$ and $Q(B(t+\varepsilon)>B(t))>0$ for all $\varepsilon>0$ and $t \in[0, \tau]$.

(ii) $Q(P(t+\varepsilon, T) \geq P(t, T))=1$ and $Q(P(t+\varepsilon, T)>P(t, T))>0$ for all $\varepsilon>0$ and $t, T \in[0, \tau]$.

Assumption 5.1 is included to simplify the subsequent analysis. It is the simplest stochastic generalization possible of a nonnegative and deterministic term structure of interest rates. Define $Z^{*}(t)$ by 


$$
\begin{aligned}
Z^{*}(t) & \equiv \frac{S\left(t, x_{d}\right)}{B(t)}+\frac{d_{1} P\left(t, T^{*}\right) 1\left(t<T^{*}\right)}{B(t)}+\frac{d_{1}}{B(t)} l\left(t \geq T^{*}\right) \\
& \equiv Z\left(t, x_{d}\right)+Z_{d}(t)
\end{aligned}
$$

where

$$
\begin{aligned}
Z\left(t, x_{d}\right) & \equiv \frac{S\left(t, x_{d}\right)}{B(t)} \\
Z_{d}(t) & \equiv \frac{d_{1}}{B(t)}\left[P\left(t, T^{*}\right) 1\left(t<T^{*}\right)\right]+1\left(t \geq T^{*}\right) \frac{d_{1}}{B\left(T^{*}\right)}
\end{aligned}
$$

Thus, the quantity $Z_{d}(t)$ corresponds to an admissible self-financing trading strategy involving $d_{1}$ bonds $P\left(t, T^{*}\right)$ held before $T^{*}$ and rolled over into the money market account after time $T^{*}$. Under the $\tilde{Q}$ defined in Sections 3 and $4, Z\left(t, x_{d}\right)$ is a square integrable $\tilde{Q}$ martingale (Proposition 3.1). Given Assumption 5.1, $Z_{d}(t)$ is bounded and

$$
\tilde{E}\left[Z_{d}(t)\right]=d_{1} \frac{P\left(0, T^{*}\right)}{B(0)}
$$

Hence, $Z_{d}(t)$ is a $\tilde{Q}$-martingale, and correspondingly $Z^{*}(t)$ is a $\tilde{Q}$-martingale.

Now consider an American call option on this new stock with a strike price $K$ and maturity date $\tau_{M}$ where $T^{*} \leq \tau_{M}$. By analogy to Section 4's definition, the arbitrage-free price at time $t$ of the American call option, $C(t)$, is defined as

$$
\frac{C(t)}{B(t)}=\underset{\theta \in \tau_{\left(t, r_{M}\right]}}{\operatorname{ess} \sup } \tilde{E}\left\{\left[\frac{S^{*}(\theta)-K}{B(\theta)}\right]^{+} \mid G_{t}\right\}
$$

Next, we turn to trading strategies which attain this value.

Definition 5.1. An $\varepsilon$-optimal early exercise strategy starting at time $t$ is a $\theta_{\varepsilon}^{t} \in$ $\tau_{\left(t, \tau_{M}\right]}$ such that for a given $\varepsilon>0$,

$$
\frac{C(t)}{B(t)} \leq \tilde{E}\left\{\left[\frac{S^{*}\left(\theta_{\varepsilon}^{t}\right)-K}{B\left(\theta_{\varepsilon}^{t}\right)}\right]^{+} \mid G_{t}\right\}+\varepsilon \quad \text { a.e. } \tilde{Q} .
$$

The early exercise strategy is said to be optimal if it is 0-optimal.

The following lemma shows that $\varepsilon$-optimal strategies exist.

Lemma 5.1 (Existence of $\varepsilon$-Optimal Strategies). $\quad\left(C(t) / B(t): t \in\left[0, \tau_{M}\right]\right)$ is a rightcontinuous $\tilde{Q}$-supermartingale, and the stopping time

$$
y_{\varepsilon}^{t} \equiv \inf \left\{s \in\left(t, \tau_{M}\right]: \frac{C(s)}{B(s)} \leq\left[\frac{S^{*}(s)-K}{B(s)}\right]^{+}+\varepsilon\right\}
$$

is $\varepsilon$-optimal for $\varepsilon>0$. 
Proof. By Theorems 1 and 2 in Fakeev (1970), there exists a right-continuous supermartingale $f(t)$ such that $f(t)$ is the minimal right-continuous supermartingale that majorizes $\max \left[\left(S^{*}(t)-K\right) / B(t), 0\right]$ and

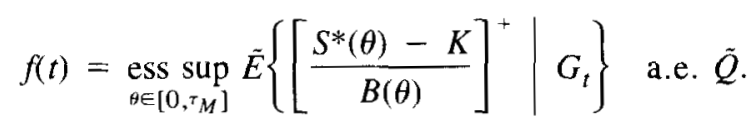

The call price $C(t)=f(t) / B(t)$, and so the first part of the lemma is established. To show $y_{\varepsilon}^{t}$ is $\varepsilon$-optimal, it suffices to show

$$
\tilde{E}\left\{\sup _{t \in\left[0, \tau^{*}\right]}\left[\frac{S^{*}(t)-K}{B(t)}\right]^{+}\right\}<\infty,
$$

because we can invoke Theorem 4 of Fakeev (1970). But

$$
\begin{aligned}
\tilde{E}\left\{\sup _{t \in\left[0, \tau_{M}\right]}\left[\frac{S^{*}(t)-K}{B(t)}\right]^{+}\right\} & \leq \tilde{E}\left\{\sup _{t \in\left[0, \tau_{M}\right]} \frac{S^{*}(t)}{B(t)}\right\} \\
& \leq \tilde{E}\left\{1+\sup _{t \in\left[0, \tau_{M}\right]}\left[\frac{S^{*}(t)}{B(t)}\right]^{2}\right\} .
\end{aligned}
$$

Applying Doob's maximal inequality, (5.9) is less than or equal to

$$
\begin{aligned}
\tilde{E}\left\{1+4\left[\frac{S^{*}\left(\tau_{M}\right)}{B\left(\tau_{M}\right)}\right]^{2}\right\} & =\tilde{E}\left\{1+4\left[\frac{S\left(\tau_{M}, x_{d}\right)}{B\left(\tau_{M}\right)}+\frac{d_{1}}{B\left(\tau_{M}\right)}\right]^{2}\right\} \\
& \leq 1+8 \tilde{E}\left\{\left[\frac{S\left(\tau_{M}, x_{d}\right)}{B\left(\tau_{M}\right)}\right]^{2}+\left(\frac{d_{1}}{B\left(\tau_{M}\right)}\right)^{2}\right\}
\end{aligned}
$$

This is finite from the square integrability of $S\left(\tau_{M}, x_{d}\right) / B\left(\tau_{M}\right)$.

Define a fictitious American contingent claim $C\left(t ; y_{\varepsilon}^{t}\right)$ to be one which has a payoff $=$ $\left[S^{*}\left(y_{\varepsilon}^{t}\right)-K\right]^{+}$at date $y_{\varepsilon}^{t}$. Given Lemma 5.1, the proof to Proposition 4.1 yields that there exists a trading strategy corresponding to this claim which requires a time $t$ investment of

$$
\tilde{E}\left\{\left[\frac{S^{*}\left(y_{\varepsilon}^{t}\right)-K}{B\left(y_{\varepsilon}^{t}\right)}\right]^{+} \mid G_{t}\right\} B(t)
$$

Hence, if the original American contingent claim traded, its value must be within $\varepsilon$ of (5.11) for all $\varepsilon>0$. However, for $\varepsilon=0$, an optimal strategy does not exist as will be shown in Proposition 5.2.

Using this analysis, (5.5) can now be given an alternative characterization. 
Proposition 5.1.

$$
C(t) / B(t)=\tilde{E}\left\{\max \left[\bar{C}\left(T^{*}\right), S^{*}\left(T^{*}\right)+d_{1}-K\right] / B\left(T^{*}\right) \mid G_{i}\right\}
$$

where

$$
\bar{C}\left(T^{*}\right)=\bar{E}\left\{\left[S^{*}\left(\tau_{M}\right)-K\right]^{+} / B\left(\tau_{M}\right) \mid G_{T^{*}}\right\} B\left(T^{*}\right)
$$

Proof. The first step is to show that the right side of (5.10) provides an upper bound for $C(t) / B(t)$. Take any $\theta \in \tau_{\left(t, \tau_{M}\right]}$. Then

$$
\begin{aligned}
\tilde{E}\left\{\left[\frac{S^{*}(\theta)-K}{B(\theta)}\right]^{+} \mid G_{t}\right\}= & \tilde{E}\left\{\left[\frac{S^{*}(\theta)-K}{B(\theta)}\right] 1_{\left(\theta<T^{*}\right)} 1_{\left(S\left(\theta, x_{d}\right)>K\right)} \mid G_{t}\right\} \\
& +\tilde{E}\left\{\left[\frac{S^{*}\left(\theta, x_{d}\right)-K}{B(\theta)}\right] 1_{\left(T^{*} \subseteq \theta<\tau_{M}\right)} 1_{\left(S\left(\theta, x_{d}\right)>K\right)} \mid G_{t}\right\} \\
& +\tilde{E}\left\{\max \left[\frac{S\left(\tau_{M}, x_{d}\right)-K}{B\left(\tau_{M}\right)}, 0\right] 1_{\left(\theta=\tau_{M}\right)} \mid G_{t}\right\} .
\end{aligned}
$$

But, $B(\theta) \geq B\left(T^{*}\right)$ on $T^{*} \leq \theta<\tau_{M}$. So using the optional sampling theorem and the law of iterated expectations, the above quantity is less than or equal to

$$
\begin{aligned}
& \tilde{E}\left\{\left[\frac{S\left(T^{*}, x_{d}\right)+d_{1}-K}{B\left(T^{*}\right)}\right] 1_{\left(\theta<T^{*}\right)} 1_{\left(S\left(\theta, x_{d}\right)>K\right)} \mid G_{t}\right\} \\
& +\tilde{E}\left\{\left[\frac{S\left(\tau_{M}, x_{d}\right)-K}{B\left(\tau_{M}\right)}\right] 1_{\left(T^{\left.* \leq \theta<\tau_{M}\right)}\right.} 1_{\left(S\left(\theta, x_{d}>K\right)\right.} \mid G_{t}\right\} \\
& +\tilde{E}\left\{\left[\frac{S\left(\tau_{M}, x_{d}\right)-K}{B\left(\tau_{M}\right)}\right]^{+} 1_{\left(\theta=\tau_{M}\right)} \mid G_{t}\right\} .
\end{aligned}
$$

The sum of the second and third terms is less than or equal to

$$
\tilde{E}\left\{\left[\frac{S\left(\tau_{M}, x_{d}\right)-K}{B\left(\tau_{M}\right)}\right]^{+} 1_{\left(T^{* \leq \theta \leq \tau_{M}}\right)} \mid G_{t}\right\}=\tilde{E}\left\{\left[\frac{\bar{C}\left(T^{*}\right)}{B\left(T^{*}\right)}\right] 1_{\left(T^{\left.* \leq \theta \leq \tau_{M}\right)}\right.} \mid G_{t}\right\} .
$$

The first term is less than or equal to

$$
\tilde{E}\left\{\left[\frac{S\left(T^{*}, x_{d}\right)+d_{1}-K}{B\left(T^{*}\right)}\right]^{+} 1_{\left(\theta<T^{*}\right)} \mid G_{t}\right\} .
$$

Therefore,

$$
C(t) / B(t) \leq \tilde{E}\left\{\max \left[\bar{C}\left(T^{*}\right), S^{*}\left(T^{*}\right)+d_{1}-K\right] / B\left(T^{*}\right) \mid G_{t}\right\}
$$


The second step is to show that there exists an $\varepsilon$-optimal strategy $y_{\varepsilon}^{t}$ such that

$$
\lim _{\varepsilon \rightarrow 0} C\left(t: y_{\varepsilon}^{t}\right)=\tilde{E}\left\{\max \left[\bar{C}\left(T^{*}\right), S^{*}\left(T^{*}\right)+d_{1}-K\right] / B\left(T^{*}\right) \mid G_{t}\right\} B(t) \quad \text { a.e. }
$$

\section{Consider}

$$
y_{\varepsilon}^{t} \equiv \begin{cases}T^{*}-\varepsilon & \text { if } S\left(T^{*}-\varepsilon, x_{d}\right)+d_{1} P\left(T^{*}-\varepsilon, \varepsilon\right)-K>\bar{C}\left(T^{*}-\varepsilon\right), \\ \tau_{M} & \text { otherwise. }\end{cases}
$$

A calculation similar to that just completed shows that

$$
\frac{C\left(t ; y_{\varepsilon}^{t}\right)}{B(t)}=\tilde{E}\left\{\max \left[\frac{S\left(T^{*}-\varepsilon, x_{d}\right)+d_{1} P\left(T^{*}-\varepsilon, \varepsilon\right)-K, \bar{C}\left(T^{*}-\varepsilon\right)}{B\left(T^{*}-\varepsilon\right)}\right] \mid G_{t}\right\} .
$$

Letting $\varepsilon \rightarrow 0$ and using the sample path continuity of our square-integrable martingales gives the result.

This proposition gives a simple method for calculating the American call's value. First, at date $T^{*}$, calculate a European call's value with exercise price $K$ and which matures at time $\tau_{M}$ (i.e., $\bar{C}\left(T^{*}\right)$ ). Second, take the minimum of this value and that obtained by exercising the American call at time $T^{*}$ (i.e., $S^{*}\left(T^{*}\right)+d_{1}-K$ ). This yields the American call's value at time $T^{*}$. Third, discount to any earlier date by using the risk neutral operator as given in $(5.10)$.

Now we are in a position to prove the main result of this section.

Proposition 5.2. Corresponding to the American call option $C(t)$, there does not exist an optimal exercise strategy $y$ with $Q\left(y>\tau_{M}\right)>0$.

Proof. Suppose there exists an optimal exercise strategy $y \in \tau_{\left[t, \tau_{M}\right]}$ with $Q\left[y<\tau_{M}\right]$ $>0$. Define

$$
y^{*}= \begin{cases}y+\frac{T^{*}-y}{2} & \text { if } t \leq y<T^{*}, \\ y+\frac{\tau_{M}-y}{2} & \text { if } T^{*} \leq y \leq \tau_{M} .\end{cases}
$$

Then $y^{*} \in \tau_{\left[t, \tau_{M}\right]}$, and $y^{*}>y$ if $y<T^{*}$, whereas $y^{*}=y$ if $y=T^{*}$. Further, let

$$
\begin{aligned}
V^{y} & =\tilde{E}\left[\left\{\left[\frac{S^{*}(y)-K}{B(y)}\right]^{+} \mid G_{t}\right\}\right] B(t) \\
V^{y *} & =\tilde{E}\left[\left\{\left[\frac{S^{*}\left(y^{*}\right)-K}{B\left(y^{*}\right)}\right]^{+} \mid G_{t}\right\}\right] B(t) .
\end{aligned}
$$

We will show that $V^{y}<V^{y *}$, which contradicts the optimality of $y$. 
Now, $S^{*}(y)=S\left(y, x_{d}\right)+d_{1} P\left(t, T^{*}\right) 1_{\left(y<T^{*}\right)}$. Hence,

$$
V^{y}(t)=\tilde{E}\left\{\left[\frac{S\left(y, x_{d}\right)}{B(y)}+\frac{d_{1} P\left(y, T^{*}\right)}{B(y)} 1_{\left(y<T^{*}\right)}-\frac{K}{B(y)}\right] 1_{\left(S^{*}(y)>K\right)} \mid G_{t}\right\} B(t) .
$$

Using the optimal stopping theorem, $S\left(t, x_{d}\right) / B(t)$ is a $\tilde{Q}$-martingale. Therefore

$$
\begin{aligned}
V^{y}(t) & =\left[\tilde{E}\left[\tilde{E}\left[\frac{S\left(y^{*}, x_{d}\right)}{B\left(y^{*}\right)}+\frac{d_{1} P\left(y, T^{*}\right)}{B(y)} 1_{\left(y<T^{*}\right)}-\frac{K}{B(y)}\right] 1_{\left(S^{*}(y)>K\right)} \mid G_{y}\right] \mid G_{t}\right] B(t) \\
& =\tilde{E}\left\{\left[\frac{S\left(y^{*}, x_{d}\right)}{B\left(y^{*}\right)}+\frac{d_{1} P\left(y, T^{*}\right)}{B(y)} 1_{\left(y<T^{*}\right)}-\frac{K}{B(y)}\right] 1_{\left(S^{*}(y)>K\right)} \mid G_{t}\right\} B(t) .
\end{aligned}
$$

From the definition of $y^{*}, 1_{\left(y<T^{*}\right)}=1_{\left(y^{*}<T^{*}\right)}$. Hence,

$$
\begin{aligned}
V^{y}(t) & =\tilde{E}\left\{\left[\frac{S\left(y^{*}, x_{d}\right)}{B\left(y^{*}\right)}+\frac{d_{1} P\left(y, T^{*}\right)}{B(y)} 1_{\left(y^{*}<T^{*}\right)}-\frac{K}{B(y)}\right] 1_{\left(S^{*}(y)>K\right)} \mid G_{t}\right\} B(t) \\
& =\tilde{E}\left\{\left[\frac{S^{*}\left(y^{*}\right)}{B\left(y^{*}\right)}-\frac{K}{B(y)}\right] 1_{\left(S^{*}(y)>K\right)} \mid G_{t}\right\} B(t) .
\end{aligned}
$$

Noting that $\tilde{Q}\left(B\left(y^{*}\right)>B(y)\right)>0, \tilde{Q}\left(B\left(y^{*}\right) \geq B(y)\right)=1$, and $\tilde{Q}(y<T)>0$, this implies that $\tilde{Q}\left(y^{*}>y\right)>0$ and $\tilde{Q}\left(y^{*} \geq y\right)=1$. Hence,

$$
V^{y}(t)<\tilde{E}\left\{\left[\frac{S^{*}\left(y^{*}\right)}{B\left(y^{*}\right)}-\frac{K}{B\left(y^{*}\right)}\right] 1_{\left(S^{*}(y)>K\right)} \mid G_{t}\right\} B(t) .
$$

But

$$
\left(\frac{S^{*}\left(y^{*}\right)-K}{B\left(y^{*}\right)}\right) 1_{\left(S^{*}(y)>K\right)} \leq\left(\frac{S^{*}\left(y^{*}\right)}{B\left(y^{*}\right)}-\frac{K}{B\left(y^{*}\right)}\right) 1_{\left(S^{*}\left(y^{*}\right)>K\right)} \text { a.e. } \tilde{Q} .
$$

This implies that

$$
V^{y}(t)<\tilde{E}\left\{\left[\frac{S^{*}\left(y^{*}\right)}{B\left(y^{*}\right)}-\frac{K}{B\left(y^{*}\right)}\right] 1_{\left(S^{*}(y)>K\right)} \mid G_{t}\right\} B(t),
$$

i.e., $V^{y}(t)<V^{y *}(t)$

This proposition proves that there does not exist an optimal exercise strategy for the American call option. The intuition for this result is straightforward. As it is suboptimal to exercise early if there are no future dividends, optimal exercise must lie in the time interval before the dividend payment date. If it is optimal to exercise early, one would like to exercise the option as close as possible to the dividend date. But given that the stock price is right-continuous and there is a discrete jump at the dividend date, the time interval over which one can exercise the option is an open interval whose supremum cannot be attained. 


\section{CONCLUSION}

This paper extends HJM (1992) to include risky assets. Various closed-form solutions for European-type options on risky assets, forward contracts, and futures contracts are provided. Secondly, it studies the pricing of American-type contingent claims on risky assets in a stochastic interest rate economy. It generalizes the previous works of HJM (1992), Bensoussan (1984), Karatzas (1988), and Roll (1977) in this regard. The analysis justifies the use of the standard procedures for calculating American contingent claim values in stochastic interest rate economies.

\section{REFERENCES}

AmIN, K., and R. JARROW (1991): "Pricing Foreign Currency Options under Stochastic Interest Rates," J. Int. Money Finance, 10(3), 310-329.

Bensoussan, A. (1984): "On the Theory of Option Pricing," Acta. Appl. Math., 2, 139-158.

BlaCK, F. (1976): "The Pricing of Commodity Contracts," J. Financial Econ., 3, 167-179.

Brennan, M. J., and E. S. Schwartz (1979): "A Continuous Time Approach to the Pricing of Bonds," J. Banking Finance, 3, 133-155.

Cox, J. C., J. E. Ingersoll, and S. A. Ross (1985): "A Theory of the Term Structure of Interest Rates," Econometrica, 53, 385-407.

DURRETT, R. (1984): Brownian Motion and Martingales in Analysis. Belmont, CA: Wadsworth.

Fakeev, A. G. (1970): "Optimal Stopping Rules for a Stochastic Process with Continuous Parameter," Theory Probab. Appl., 15, 324-331.

HaRRison, J. M., and D. M. KRePs (1979): "Martingales and Arbitrage in Multiperiod Security Markets," J. Econ. Theory, 20, 381-408.

Harrison, J. M., and S. R. Pliska (1981): "Martingales and Stochastic Integrals in the Theory of Continuous Trading," Stoch. Processes Appl., 11, 215-260.

Heath, D. C., R. A. Jarrow, and A. J. Morton (1992): "Bond Pricing and the Term Structure of Interest Rates: A New Methodology for Contingent Claims Valuation," Econometrica, 60(1), $77-105$.

Jarrow, R. (1987): "The Pricing of Commodity Options with Stochastic Interest Rates," Adv. Futures Options Res. 2, 19-45.

Karatzas, I. (1988): "On the Pricing of American Options," Appl. Math. Optim., 17, 37-60.

Karatzas, I., and S. Shreve (1988): Brownian Motion and Stochastic Calculus. New York: Springer-Verlag.

LIPTSER, R. S., and A. N. Shiryayev (1977): Statistics of Random Processes. I: General Theory. New York: Springer-Verlag.

Merton, R. C. (1973): "The Theory of Rational Option Pricing," Bell J. Econ. Management Sci., 4, 141-183.

Morton, A. J. (1989): “Arbitrage and Martingales," Ph.D. thesis, School of Operations Research, Cornell University.

RoLl, R. (1977): “An Analytic Formula for Unprotected American Call Options on Stocks with Known Dividends," J. Financial Econ., 5, 251-258. 